\title{
Application of efficient generator-coordinate subspace-selection algorithm to neutrinoless double- $\beta$ decay
}

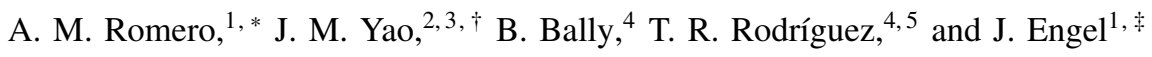 \\ ${ }^{1}$ Department of Physics and Astronomy, University of North Carolina, Chapel Hill, North Carolina 27516-3255, USA \\ ${ }^{2}$ School of Physics and Astronomy, Sun Yat-sen University, Zhuhai 519082, P.R. China \\ ${ }^{3}$ Facility for Rare Isotope Beams, Michigan State University, East Lansing, Michigan 48824-1321, USA \\ ${ }^{4}$ Departamento de Física Teórica y Universidad Autónoma de Madrid, E-28049 Madrid, Spain \\ ${ }^{5}$ Centro de Investigación Avanzada en Física Fundamental-CIAFF-UAM, E-28049 Madrid, Spain
}

(Dated: June 22, 2021)

\begin{abstract}
The generator coordinate method begins with the variational construction of a set of non-orthogonal meanfield states that span a subspace of the full many-body Hilbert space. These states are then often projected onto states with good quantum numbers to restore symmetries, leading to a set with members that can be similar to one another, and it is sometimes possible to reduce this set without greatly affecting results. Here we propose a greedy algorithm that we call the energy-transition-orthogonality procedure (ENTROP) to select subsets of important states. As applied here, the approach selects on the basis of diagonal energy, orthogonality, and contribution to the matrix element that governs neutrinoless double- $\beta$ decay. We present both shell-model and preliminary ab initio calculations of this matrix element for the decay of ${ }^{76} \mathrm{Ge}$, with quadrupole deformation parameters and the isoscalar pairing strength as generator coordinates. ENTROP converges quickly, reducing significantly the number of basis states needed for an accurate calculation.
\end{abstract}

\section{INTRODUCTION}

The observation of neutrinoless double- $\beta(0 v \beta \beta)$ decay, in which two protons decay into two neutrons without neutrino emission, would show that neutrinos are Majorana particles. The half life for such a decay depends on the nuclear matrix element (NME) of the transition operator between the groundstates of the initial and final nuclei. The NME, which must be computed, is model-dependent, with results differing from model to model by factors of up to three. Reducing the discrepancy is difficult because each model has its own uncontrolled approximations. One way forward is to use $a b$ initio methods to compute the NME from first principles. In particular, in-medium similarity renormalization group (IMSRG) methods [1, 2] with chiral interactions are promising and have already been applied to nuclei such as ${ }^{48} \mathrm{Ca}\left[3\right.$, 4] and ${ }^{76} \mathrm{Ge}$ [4] that are of great interest to experimentalists. The approach leads to effective Hamiltonians and transition operators to be used together with traditional many-body methods that cannot by themselves easily incorporate high-energy correlations. With the generator coordinate method (GCM) [5] as the traditional one, the approach has proved successful in describing the spectra of low-lying states, and has been used to compute the NME for $0 v \beta \beta$ decay of ${ }^{48} \mathrm{Ca}[3,6]$.

The GCM, which has been applied most often within nuclear energy-density-functional theory [7--10], provides an effective way to construct wave functions that include collective correlations. Such correlations, in particular involving deformation (both axial and triaxial [11]), and pairing (of both likeparticle and proton-neutron [12, 13] type), are important for $0 v \beta \beta$ NMEs. The GCM incorporates the effects of these degrees of freedom by taking them as "generator coordinates," with values on a mesh that approximates the continuum. Unfortunately, the method scales exponentially with the number of coordinates. Including many mesh points leads to a large set of non-orthogonal states and a significant computational burden. Some of these basis states, however, may closely resemble others or have little representation in low-lying wave functions, and can therefore be omitted. Here, we propose a schemes that we call the energy-transition-orthogonality procedure (ENTROP) for rejecting unimportant states. As the name suggests, the approach is designed to work for transition matrix elements and we apply it to $0 v \beta \beta$ NMEs.

The particular case that we examine is the decay of ${ }^{76} \mathrm{Ge}$ to ${ }^{76} \mathrm{Se}$. Both nuclei exhibit triaxial deformation [14, 15]. If the GCM includes two deformation coordinates and one that represents the effects of isoscalar pairing, [11, 13] in a large single-particle space, the computing time required to restore all the broken symmetries in the resulting set of states can be significant, making the three-coordinate case a good one for testing/applying our algorithms. We do so within two kinds of calculations, the first in a small shell-model valence space and an appropriate semi-phenomenological interaction, and the second in seven major shells and an ab initio interaction resulting from the in-medium evolution of a chiral Hamiltonian.

The rest of this paper is organized as follows: Section II discusses the nature of the GCM basis and presents ENTROP, along with a procedure based on the work of Ref. [16]. In Section III we present the results obtained after the application of these methods in the computations just described. In Section IV, we offer conclusions.

\section{METHODS}

$M_{0 v}$, the NME that we wish to calculate enters the rate of $0 v \beta \beta$ decay that is mediated by the exchange of light Majorana 
neutrinos as follows:

$$
\left[T_{1 / 2}^{0 v}\right]^{-1}=G_{0 v}(Q, Z)\left|M_{0 v}\right|^{2}\left|\sum_{k} m_{k} U_{e k}^{2}\right|^{2},
$$

where $Q$ is the energy difference between the initial and final atoms, $G_{0 v}$ is a phase space factor, the $m_{k}$ are the masses of the three light neutrinos and the $U_{e k}$ are the elements of the neutrino mixing matrix that connects the electron neutrino to the state with mass eigenvalue $m_{k}$. One traditionally separates $M_{0 v}$ into Gamow-Teller, Fermi, and tensor pieces,

$$
M_{0 v}=M_{0 v}^{G T}-\frac{g_{V}^{2}}{g_{A}^{2}} M_{0 v}^{F}+M_{0 v}^{T},
$$

where $g_{V}$ and $g_{A}$ are the nuclear vector and axial-vector weak coupling constants (we use $g_{A}=1.27$ here) and $M_{0 v}^{G T}, M_{0 v}^{F}$, and $M_{0 v}^{T}$ are defined, e.g., in Ref. [17] (though $M_{0 v}^{F}$ mistakenly contains an extra factor of $g_{V}^{2} / g_{A}^{2}$ there.)

The GCM combines constrained mean-field states into a fully-correlated nuclear wavefunction [18], which we call a GCM state from now on. The starting point is the set of meanfield states, for us Hartree-Fock-Bogoliubov (HFB) quasiparticle vacua $|\varphi(\boldsymbol{q})\rangle$, that minimize the energy $\langle\varphi(\boldsymbol{q})|H| \varphi(\boldsymbol{q})\rangle$ under the constraint that a vector of observables $\hat{Q}=$ $\left(\hat{Q}_{1}, \hat{Q}_{2}, \ldots, \hat{Q}_{N}\right)$ takes the values $\langle\varphi(\boldsymbol{q})|\hat{\boldsymbol{Q}}| \varphi(\boldsymbol{q})\rangle=\boldsymbol{q}$. The coordinates $\boldsymbol{q}$ that label the mean-field states are frequently chosen to lie on an $\mathrm{N}$-dimensional mesh that discretizes the space of quasiparticle vacua. The coordinate operators $\hat{Q}$ are generally those that are important for a good description of the nucleus. In this paper, we choose the quadrupole operators, $\hat{Q}_{20}$ and $\hat{Q}_{22}$, and the isoscalar-pair creation operator $\hat{P}_{0}^{+}$ as generator coordinates. Details appear in the next section.

A GCM state $\left|\Psi_{N Z}^{J M}\right\rangle$ has the form

$$
\left|\Psi_{N Z}^{J M}\right\rangle=\sum_{K, \boldsymbol{q}} f_{K, \boldsymbol{q}}^{J}|N Z J M K, \boldsymbol{q}\rangle,
$$

where the states $|N Z J M K, \boldsymbol{q}\rangle$ are projections of the $|\varphi(\boldsymbol{q})\rangle$ :

$$
|N Z J M K, \boldsymbol{q}\rangle=\hat{P}_{M K}^{J} \hat{P}^{N} \hat{P}^{Z}|\varphi(\boldsymbol{q})\rangle .
$$

Here $\hat{P}_{M K}^{J}$ is the operator that projects a state onto components with well defined angular momentum $J, z$-projection $M$, and intrinsic-z-projection $K$. Since $K$ is not a good quantum number for a triaxially-deformed nucleus, components with all values of $K$ contribute to a GCM state (through " $K$ mixing"). The operators $\hat{P}^{N}$ and $\hat{P}^{Z}$ project states onto components with well-defined neutron number $N$ and proton number $Z$.

The projection operators produce basis states that are not orthonormal, and lead to the Hill-Wheeler-Griffin (HWG) equation for $f_{K, q}^{J}$,

$$
\sum_{K^{\prime}, \boldsymbol{q}^{\prime}}\left[\mathcal{H}_{K K^{\prime}}^{J}\left(\boldsymbol{q}, \boldsymbol{q}^{\prime}\right)-E^{J} \mathcal{N}_{K K^{\prime}}^{J}\left(\boldsymbol{q}, \boldsymbol{q}^{\prime}\right)\right] f_{K^{\prime}, \boldsymbol{q}^{\prime}}^{J}=0,
$$

where the Hamiltonian and norm kernels $\mathcal{H}$ and $\mathcal{N}$ are given by the expressions

$$
\begin{aligned}
& \mathcal{H}_{K K^{\prime}}^{J}\left(\boldsymbol{q}, \boldsymbol{q}^{\prime}\right)=\left\langle N Z J M K, \boldsymbol{q}|\hat{H}| N Z J M K^{\prime}, \boldsymbol{q}^{\prime}\right\rangle \\
& \mathcal{N}_{K K^{\prime}}^{J}\left(\boldsymbol{q}, \boldsymbol{q}^{\prime}\right)=\left\langle N Z J M K, \boldsymbol{q} \mid N Z J M K^{\prime}, \boldsymbol{q}^{\prime}\right\rangle,
\end{aligned}
$$

and $E^{J}$ is the energy of the state with angular momentum $J$ that we are interested in (we've suppressed the labels $N$ and $Z$ in places for convenience). We solve the HWG equation in the standard way [18], by diagonalizing the norm kernel to obtain a basis of "natural states" and then diagonalizing the Hamiltonian $H$ in that basis. The second diagonalization can be numerically unstable, a problem we deal with by truncating the natural basis to include only states with norm eigenvalues larger than a reasonable value. That step eliminates the instability by removing states that are nearly linearly dependent on others.

The computational time in this method lies mostly in the construction of the kernels for the Hamiltonian and $0 v \beta \beta$ transition operators. That process entails an integration of matrix elements of two-body operators over Euler and gauge angles to project onto conserved quantities. The norm kernels require the same integration, though without an operator sandwiched between states. It is difficult to know ahead of time how dense to make the coordinate mesh or how far to extend it, and so we would like to select a subset of points on the mesh before computing all the kernels. We can expect some basis states to contribute little to the energy of the GCM ground state or to the $0 v \beta \beta$ NME between two GCM states, and others to be very similar to one another (the result of too dense a mesh). Our best prescription for subset selection is based on three observations:

- States with lower expectation values for the Hamiltonian are in general more important than those with higher expectation values.

- The largest contributions to NMEs often come from transitions between basis states (in our case in two different nuclei) with the same values for the collective coordinates $q$ [7, 19].

- States that can nearly be represented as a linear combination of states in the selected subset need not themselves be included in the subset. They add only numerical noise to the HWG equation that must be removed in its solution.

ENTROP incorporates these observations through the following procedure: we order the $|\varphi(\boldsymbol{q})\rangle$ in each nucleus by diagonal energies $\langle H\rangle_{J K q} \equiv \mathcal{H}_{K K}^{J}(\boldsymbol{q}, \boldsymbol{q}) / \mathcal{N}_{K K}^{J}(\boldsymbol{q}, \boldsymbol{q})$ and select the one with the lowest value in, e.g., the initial nucleus. We then move to the final nucleus, selecting first the state with the lowest diagonal energy and then the state with the same coordinates $\boldsymbol{q}$ as the first state from the initial nucleus (the "partner" of that state), provided that its projection onto the previously included state has squared length $L$ less than some cutoff value $L_{c}$ (so that it is nearly linearly independent). Next we return to the initial nucleus,selecting the state with the secondlowest diagonal energy and the partner of the first the state in the final nucleus, again after checking projections. We continue in this way, including each state that we examine only if its projection onto the space of previously-selected states has length less than $L_{c}$, i.e. if

$$
L \equiv \frac{\left\langle n+1\left|P^{(n)}\right| n+1\right\rangle}{\langle n+1 \mid n+1\rangle}<L_{c} .
$$




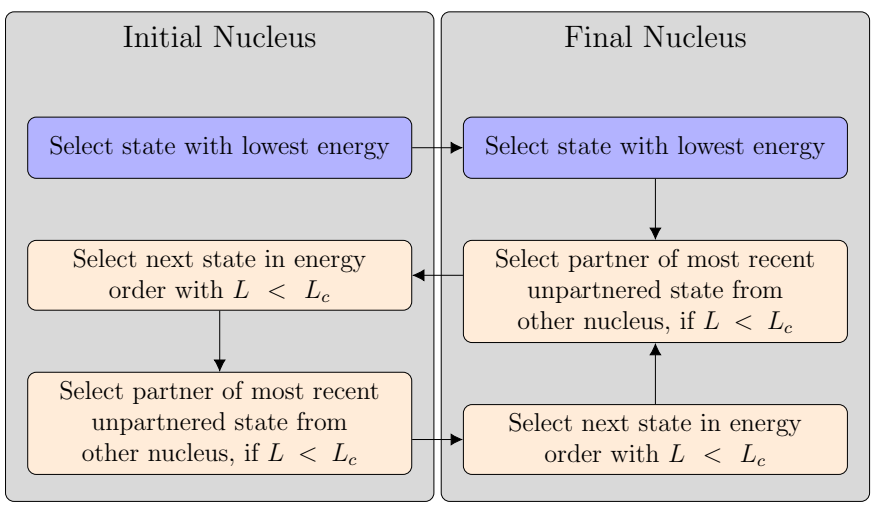

FIG. 1. Schematic diagram of the state selection order produced by ENTROP.

Here $|n+1\rangle$ is the state we are testing and $P^{(n)}$ is the projector onto the $n$ states already selected (see the Appendix for details). After including each new state we diagonalize $H$ in the appropriate subset and look for convergence of the eigenvalues and NME. Fig. 1 contains a flow chart representing the selection procedure. The method saves time because we compute the off-diagonal norm kernels only of the states we examine and the off-diagonal Hamiltonian and $0 v \beta \beta$ kernels only of the states we eventually select.

The procedure just outlined contains the parameter $L_{c}$, the value of which we have yet to specify. To determine it, we repeat the entire procedure for a range of $L_{c}$ and within several pairs of small subspaces of the full space (one space in the pair for the initial nucleus and one for the final). We then choose the smallest value of $L_{c}$ that "works" within each pair of subspaces - that is, a value that brings us so close to the energies and NMEs obtained in each complete subspace pair that increasing $L_{c}$ further (and thus including more basis states) has little effect. We then assume that the same will be true in any subspace pair, including one that contains all basis states on the mesh in both nuclei. This assumption cannot be rigorously justified but is reasonable.

Our original intent was to implement something like the procedure discussed in Ref. [16], which successfully reproduces the low-lying portions of collective spectra within energy-density functional theory. In that approach, one starts from random mean-field states (Slater determinants in Ref. [16] itself) obtained without constraints, descending towards local minima in the energy surface via imaginary-time evolution and selecting states along the way to subject to an orthogonality test like the one described here. We test a modification of that procedure, in which we use gradient descent rather than imaginary-time evolution to approach energy minima in our space of quasiparticle vacua, for the decay of ${ }^{76} \mathrm{Ge}$ to ${ }^{76} \mathrm{Se}$ with the shell-model space and Hamiltonian described at the beginning of the next section. We use 50 randomly selected quasiparticle vacua as starting points, and then select a random number of states along the corresponding paths of descent once the energy has dropped below $10 \mathrm{MeV}$. In the most successful version of this procedure, we then order the states by energy and fix a cutoff $L_{c}$ in the same way as with

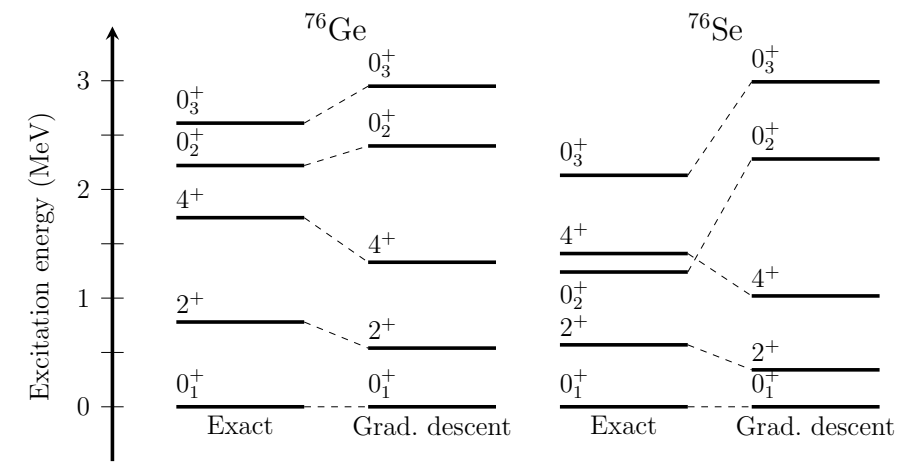

FIG. 2. Low-lying energy spectrum of ${ }^{76} \mathrm{Ge}$ and ${ }^{76} \mathrm{Se}$ computed by shell-model code BIGSTICK [20, 21] (Exact) and from the procedure based on that in the Ref. [16] and described in the text (Grad. descent). The figure doesn't show an overall upward shift in the Grad.descent energies of about $1.5 \mathrm{MeV}$ in ${ }^{76} \mathrm{Ge}$ and $2 \mathrm{MeV}$ in ${ }^{76} \mathrm{Se}$.

ENTROP. But while we can roughly reproduce the exact spectra of ${ }^{76} \mathrm{Ge}$ and ${ }^{76} \mathrm{Se}$ with about 30 states in each nucleus (from about 17 distinct starting points in ${ }^{76} \mathrm{Ge}$ and 18 in ${ }^{76} \mathrm{Se}$ ), as we show in Fig. 2, we are not able to obtain as accurate an NME as we can with ENTROP (see Fig. 4). That result is not entirely surprising because, unlike the GCM, the procedure of Ref. [16] in no way ensures that states in one nucleus are similar to those in the other.

\section{RESULTS}

\section{A. Shell-model test}

To test the accuracy of ENTROP, we examine the decay ${ }^{76} \mathrm{Ge} \longrightarrow{ }^{76} \mathrm{Se}$ in a model space built on the $0 f_{5 / 2}, 1 p_{3 / 2}, 1 p_{1 / 2}$ and $0 g_{9 / 2}$ orbits, with the effective valence-space shell-model Hamiltonian GCN2850 [22]. The model space allows an exact solution with modern shell model codes. Ref. [11] carefully examined the performance of the GCM for this problem, constructing a mesh of 184 quasiparticle vacua with constraints on the coordinates representing axial deformation, triaxiality, and the isoscalar pairing strength. The operators that correspond to these coordinates are

$$
\begin{aligned}
\hat{Q}_{20} & =\sum_{i} r_{i}^{2} Y_{i}^{20} \\
\hat{Q}_{22} & =\sum_{i} r_{i}^{2} Y_{i}^{22} \\
\hat{P}_{0} & =\frac{1}{2 \sqrt{2}} \sum_{l, \alpha} \sqrt{2 l+1}\left[a_{l, \alpha}^{\dagger} a_{l, \alpha}^{\dagger}\right]_{M=0, T_{z}=0}^{J=1, T=0}+\text { h.c. },
\end{aligned}
$$

where $i$ labels nucleons in first quantization, the square brackets signify the coupling of orbital angular momentum, spin, and isospin, and the operator $a_{l, \alpha}^{\dagger}$ creates a particle in the single-particle level with orbital angular momentum $l$ and other quantum numbers specified by $\alpha$. Here we replicate the calculation of Ref. [11] to test the results of restricting ourselves to particular subsets of its states. To construct the basis 


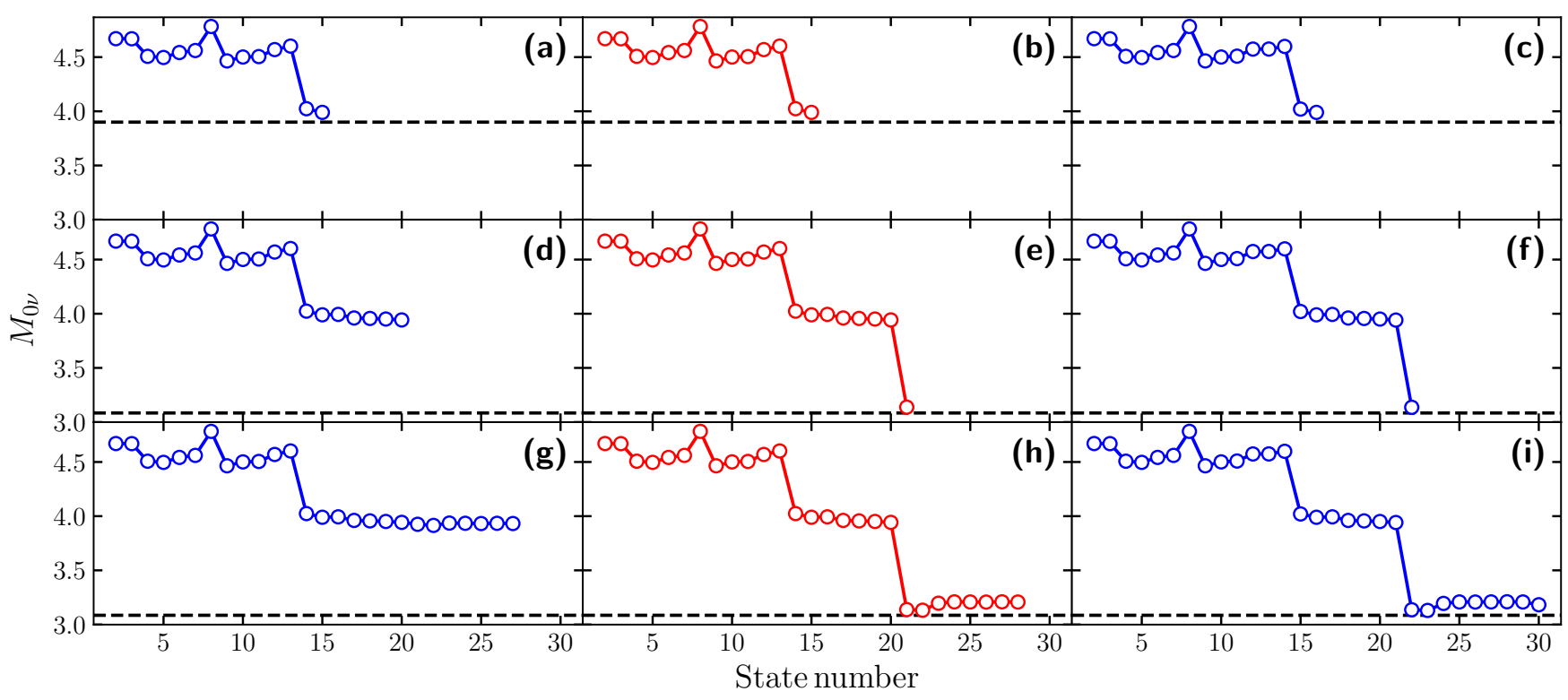

FIG. 3. Valence-space NME for the decay of ${ }^{76} \mathrm{Ge}$, computed with the GCN2850 interaction in pairs of subspaces spanned together by the first 20, 40 and 60 states (top row, middle row, and bottom row) after applying ENTROP up to the combined (from both-nuclei) state number indicated by the $x$ axis. The states are ordered as indicated in Fig. 1. with cutoff values $L_{c}$ of 0.994 (left column), 0.995 (middle column), and 0.996 (right column). The dashed line is the result produced by the full set of states in each subspace pair. The value $L_{c}=0.995$, corresponding to the middle column (in red) is the smallest that reproduces the full results in all three subspace pairs.

states and solve the resulting eigenvalue problem, we use the FORTRAN program TAURUS [23, 24]. Fig. 2] shows the lowlying spectra produced by the 184-state GCM and the method related to that of Ref. [16]. As mentioned in the methodology section, in order to choose the cutoff $L_{c}$, we evaluate the NME in subspace pairs with increasing dimension, here those spanned by the first 20, 40, and 60 states chosen in the order indicated in Fig. 1] with $L_{c}$ set to 1 to make sure no states are skipped. We then find that $L_{c}=0.995$ (so that trial states have to be almost completely expressible in terms of those already selected to be rejected) is the smallest value that accurately allows us to reproduce the NME in all three subspaces.

Fig. 3 shows how well the cutoff $L_{c}=0.995$ works for the NME in the subspaces just mentioned. In all three cases it yields a number very close to the complete ones, with little more than half the basis states in the two larger subspace pairs. This analysis leads us to expect that the states we will discard with $L_{c}=0.995$ in our complete calculation so nearly lie in the spaces spanned by the states we will have already selected that they will not alter the results.

Our expectation turns out to be the case. Fig. 4 shows the results of our analysis in panel (b); after 20 states, the NME is very close to the full GCM value. Panel (a) in the same figure shows what happens when we do not use the $0 v \beta \beta$ operator to select states, that is, when we do not include partner states. Performance is generally worse, and even after 60 states the result is not as close to the full one as it is after 20 states in panel (b). Finally, panel (c) shows the result of the Ref. [16]like analysis discussed in the previous section. As we noted there, our NME does not approach the exact result within the set of states we collect.
The convergence of the ground-state energies under ENTROP behaves a little differently than that of the NME. Figs. 5 and 6 show the convergence towards the ground-state energies of ${ }^{76} \mathrm{Ge}$ and ${ }^{76} \mathrm{Se}$, respectively, within ENTROP and in the full-GCM "natural basis", the one that for a given number of states picks out the subspace that most closely spans the full set [25]. Even after the very first state - the unconstrained HFB minimum, the ENTROP energy is well within a percent of the correct one. After that it converges more gradually, eventually tracking the results of the natural-basis truncation. Using a larger value of $L_{c}$ than 0.995 simply extends the ENTROP curve along that corresponding to the natural basis. We believe that this is the best that one can do without an explicit (and time consuming) consideration of off-diagonal contributions to the energy. Fortunately, however, the long tail of rejected states makes almost no difference in the NME; if we extend the curves in the top two panels of Fig. 4 the NME never moves significantly from the full GCM value.

\section{B. Ab initio calculation}

We turn now to the ab initio computation of the same decay NME. Using a chiral NN $+3 \mathrm{~N}$ interaction [26, 27] employed in recent studies of light nuclei [28] and ${ }^{48} \mathrm{Ca}$ [3], and evolving it and the decay operator according to the equations of the IMSRG [1] with a reference ensemble comprising prolate, spherical, and oblate HFB minima in both ${ }^{76} \mathrm{Ge}$ and ${ }^{76} \mathrm{Se}$ and with $e_{\max }=6$ (i.e. in 7 shells), we repeat the steps just described. Results in a larger space will be published soon. Unlike in our shell-model computation - and this would be the case in 
any realistic application — we do not have "complete" results with which to test our approximations. Our mesh in the space of deformation parameters $\beta, \gamma$, and $\varphi$ [11] (related to the axial deformation, triaxiality and isoscalar pairing strength used in the shell-model calculation) contains 145 points (or 290 if we count the points in both nuclei), and a complete solution to the HWG equation in the resulting space is more than we can currently handle. We thus once again apply ENTROP, this time without comparing to an exact result.

Fig. 7 shows that within subspace pairs consisting of 20, 30, and 40 total states, a cutoff value $L_{c}=0.902$ is sufficient to obtain the correct NME for each pair. It is the smallest value of the cutoff that does so. We therefore adopt this cutoff and generate another sequence of states, leading to the results in Figs. 8 and 9. Though the energies in Fig. 9 are still falling slowly after 18 and 16 states in ${ }^{76} \mathrm{Ge}$ and ${ }^{76} \mathrm{Se}$, the NME in Fig. 8 has more or less converged long before, by about 20 states from the two nuclei combined. Of course, we cannot be sure that the long plateau continues indefinitely, but the longer it extends, the more confidence we have.

The three parts of our NME are

$$
\begin{aligned}
M_{0 v}^{G T} & =2.68 \\
-\frac{g_{V}^{2}}{g_{A}^{2}} M_{0 v}^{F} & =0.65 \\
M_{0 v}^{T} & =-0.16 .
\end{aligned}
$$

A recent valence-space IMSRG calculation obtained $M_{0 v}^{G T}=$ 2.76, $g_{V}^{2} / g_{A}^{2} M_{0 v}^{F}=0.54$, and $M_{0 v}^{T}=-0.49$ with the same chiral interaction and the same value of $e_{\max }$ [4]. The main difference between the two sets of results is in the tensor matrix element. In the valence-space calculation, this component reduces the total NME by $17 \%$, while in ours it reduces it by

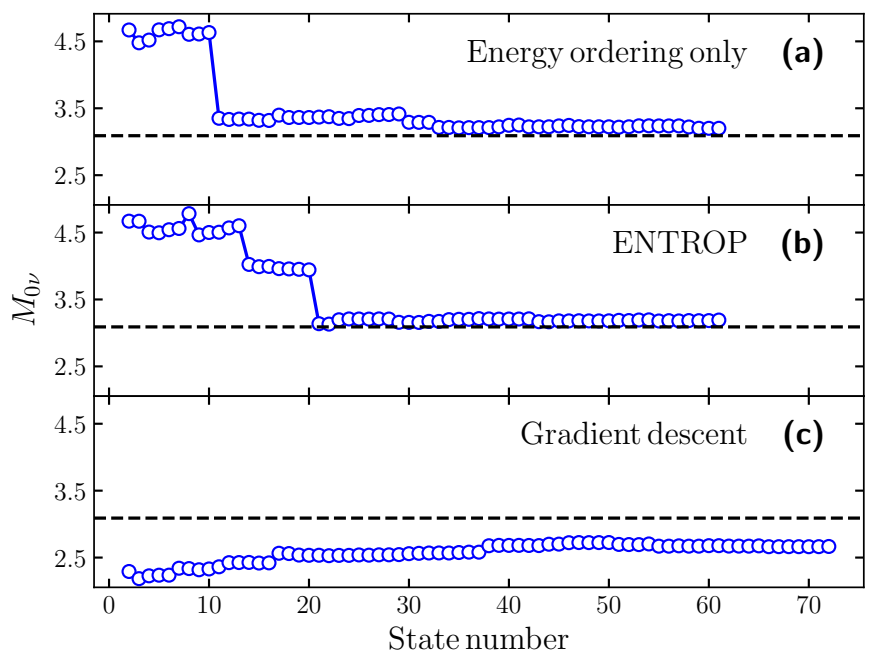

FIG. 4. Valence-space NME for the decay of ${ }^{76} \mathrm{Ge}$ produced by ENTROP without including "partner states" (top, see text), by full ENTROP with $L_{c}=0.995$ (middle), and by the procedure based on that in Ref. [16] (bottom), at the combined (both-nuclei) state number indicated by the $x$ axis.

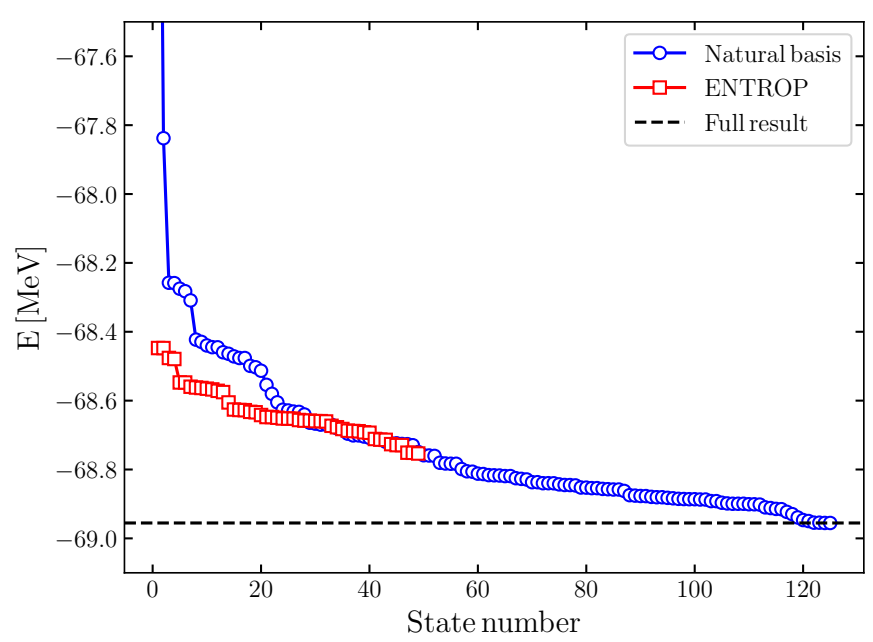

FIG. 5. Valence-space ${ }^{76} \mathrm{Ge}$ ground-state energy in the natural basis (blue) and from ENTROP (red). The dashed line is the full GCM result. Here, the state number refers to a single nucleus only.

only $5 \%$, a number that is similar to what has been obtained in more phenomenological computations. We will publish a more complete calculation of these matrix elements with a larger value for $e_{\max }$ elsewhere.

\section{CONCLUSIONS}

We have presented a greedy algorithm called ENTROP to select the most important mean-field states for GCM calculations of ground-state energies and $0 v \beta \beta$ NMEs. The algorithm starts with one HFB quasiparticle vacuum per point in a large mesh within a space of collective coordinates, and reduces the number of projected HFB states that need to be worked with. The steps in the procedure, briefly, are as follows:

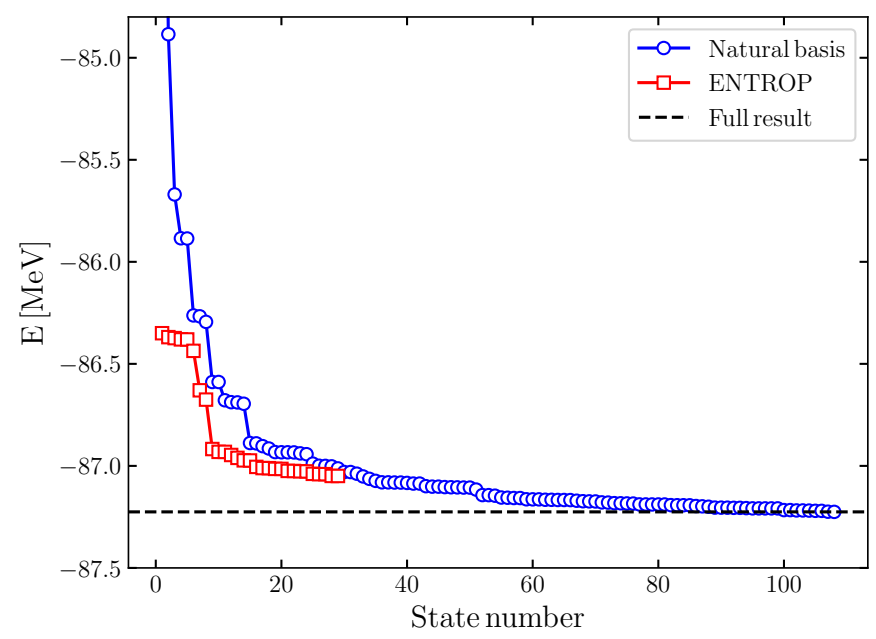

FIG. 6. Same as Fig. 5 but for ${ }^{76} \mathrm{Se}$. 


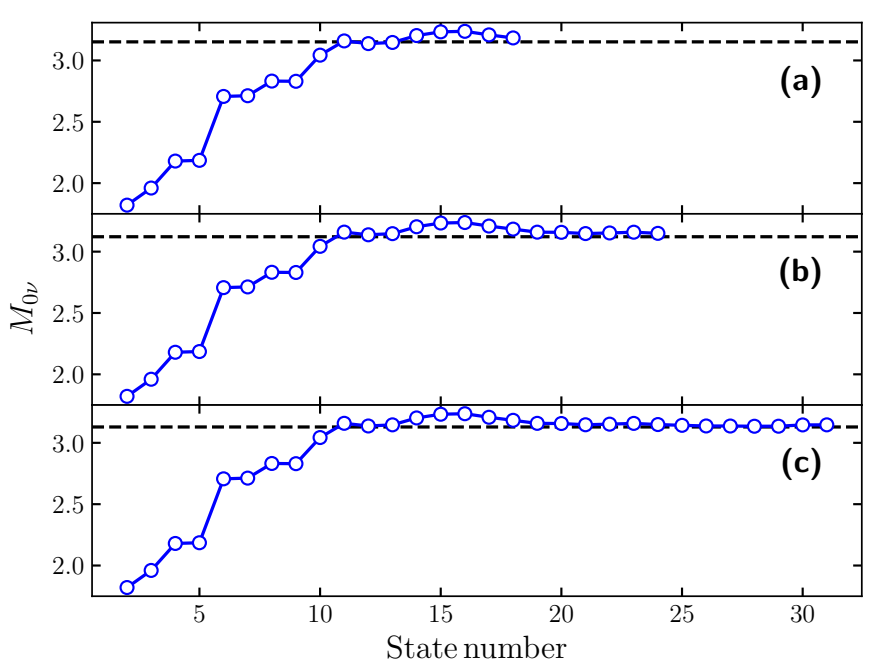

FIG. 7. Ab initio NME for the decay of ${ }^{76} \mathrm{Ge}$, with the total number of states in the subset pairs equal to 20 (top), 30 (middle), and 40 (bottom), after applying ENTROP up to the combined (both-nuclei) state number indicated by the $x$ axis.

- Sort the projected states by their diagonal energies.

- Consider the first $N$ states in each nucleus for several values of $N$.

- Find the smallest value of $L_{c}$ that, when the selection scheme in Fig. 11 is applied, leads to subsets of the first $N$ states (for all the values of $N$ ) that succeed in reproducing the corresponding NME.

- Use that value of $L_{c}$ to create a subspace pair in the full GCM spaces, solve the corresponding HWG equations, and compute the NME.

The scheme reduces computational effort because we need

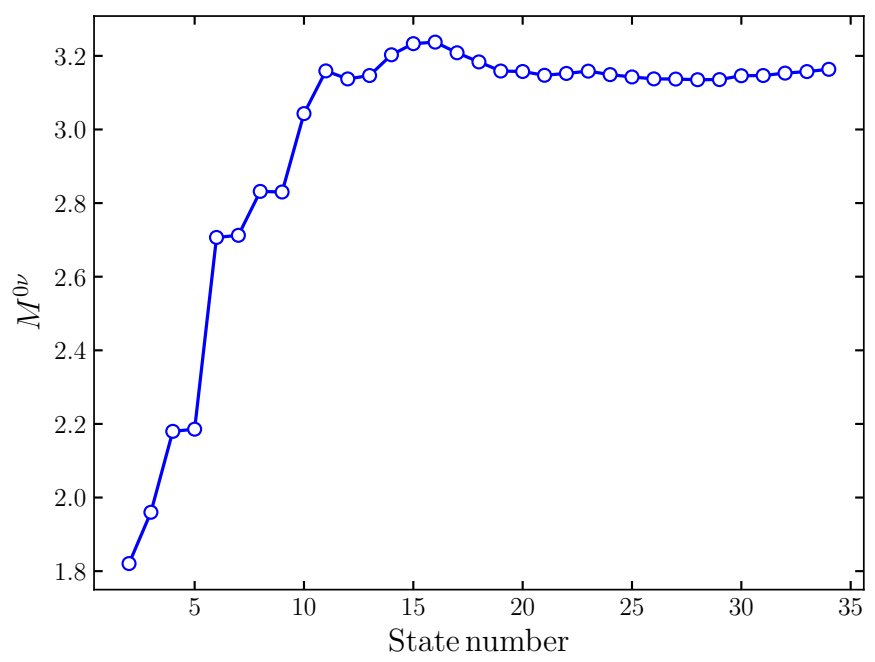

FIG. 8. ENTROP NME for the decay of ${ }^{76} \mathrm{Ge}$, with $L_{c}=0.902$.

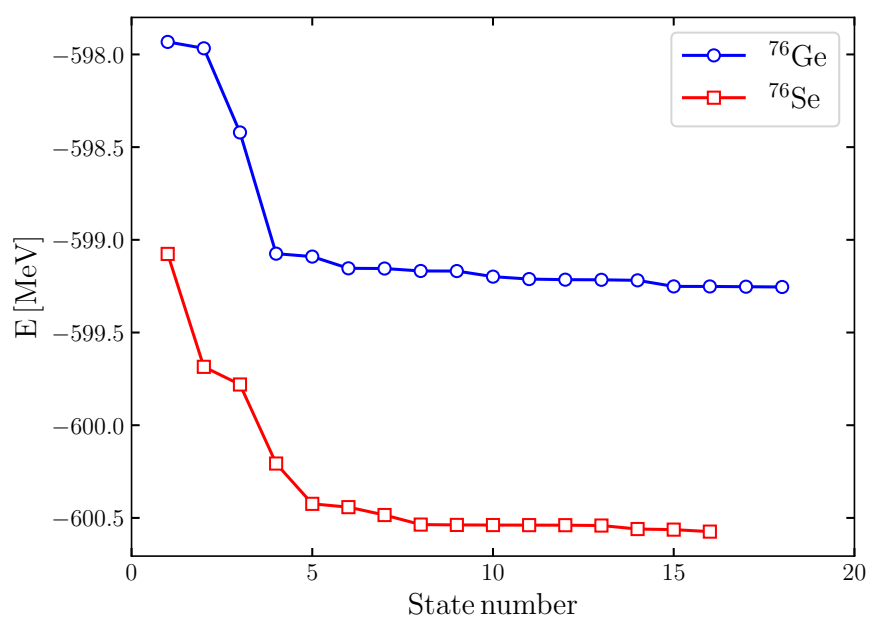

FIG. 9. Ab initio ENTROP ground-state energies for ${ }^{76} \mathrm{Ge}$ (blue) and ${ }^{76} \mathrm{Se}$ (red), with $L_{c}=0.902$. The state number refers to a single nucleus only.

to compute norm kernels only for the projected states states that we test, and Hamiltonian and $\beta \beta$ kernels only for those that are actually selected. We successfully tested our method in a computation of the NME for the decay of ${ }^{76} \mathrm{Ge}$ within a valence shell-model space with a phenomenological interaction; it reduced computation time there by more than a factor of 100. We also applied the method to an ab initio computation of the same NME with an IMSRG-evolved chiral interaction, where a full calculation is too time consuming to carry out. In both our examples, ENTROP appears to lead to a suitable basis with many fewer states than in typical GCM calculations, opening up the possibility of adding new generator coordinates to the usual set.

As we just noted, ENTROP requires norm kernels for the set of states that are tested, and although those take less time to compute than do Hamiltonian or $\beta \beta$ kernels, they are still not always cheap. We have found the use of approximate norm kernels, e.g. from unprojected basis states to be promising, and are also exploring machine-learning techniques to reduce the number of norm kernels that must be calculated.

\section{ACKNOWLEDGMENTS}

We thank H. Hergert, C.F. Jiao, and R. Wirth for fruitful discussions, and A. Belley for sending us results of VS-IMSRG calculations. This work is supported in part by the U.S. Department of Energy, Office of Science, Office of Nuclear Physics under Awards No. DE-SC0017887, No. DE-FG0297ER41019, No. DE-SC0015376 (the DBD Topical Theory Collaboration) and No. DE-SC0018083 (NUCLEI SciDAC-4 Collaboration). It is also supported by the Spanish Ministerio de Ciencia e Innovación under contract PGC2018-094583-BI00 and the European Union's Horizon 2020 research and innovation programme under the Marie Skłodowska-Curie grant agreement No. 839847. Computing resources were provided 
by the Institute for Cyber-Enabled Research at Michigan State University, the Research Computing group at the University of North Carolina, and the U.S. National Energy Research Scientific Computing Center (NERSC), a DOE Office of Science User Facility supported by the Office of Science of the U.S. Department of Energy under Contract No. DE-AC02$05 \mathrm{CH} 11231$.

\section{Appendix: Squared length of projection onto a subspace}

To compute $L$ for a given state and a subspace of previously selected states we proceed as follows. Let the subspace be spanned by the un-normalized and non-orthogonal vectors $|1\rangle,|2\rangle, \ldots,|n\rangle$. A candidate state $|n+1\rangle$ will not be included in this set if it is nearly a linear superposition of those states. Calling the projector onto the subspace $P^{(n)}$, we have

$$
P^{(n)}|n+1\rangle=\sum_{i=1}^{n} \alpha_{i}^{(n)}|i\rangle
$$

for some coefficients $\alpha_{i}^{(n)}$, which are determined by requiring that $|n+1\rangle-P^{(n)}|n+1\rangle$ is orthogonal to $|k\rangle$ for all $k \leq n$,

$$
\langle k \mid n+1\rangle-\left\langle k\left|P^{(n)}\right| n+1\right\rangle=0 \longrightarrow \sum_{i=1}^{n}\langle k \mid i\rangle \alpha_{i}^{(n)}=\langle k \mid n+1\rangle .
$$

In matrix form, Eq. A.2 is

$$
\boldsymbol{S}^{(n)} \boldsymbol{\alpha}^{(n)}=\boldsymbol{\beta}^{(n)}
$$

with $S_{i j}^{(n)}=\langle i \mid j\rangle$ and $\beta_{i}^{(n)}=\langle i \mid n+1\rangle$. The solution is

$$
\boldsymbol{\alpha}^{(n)}=\left(\boldsymbol{S}^{(\boldsymbol{n})}\right)^{-1} \boldsymbol{\beta}^{(\boldsymbol{n})} .
$$

The squared length $L$ of the projection of the normalized candidate state onto the space spanned by the already selected states is then

$$
\begin{aligned}
L & =\frac{\left\langle n+1\left|P^{(n)}\right| n+1\right\rangle}{\langle n+1 \mid n+1\rangle}=\frac{\sum_{i=1}^{n}\langle n+1 \mid i\rangle \alpha_{i}^{(n)}}{\langle n+1 \mid n+1\rangle} \\
& =\frac{\boldsymbol{\beta}^{(n) \dagger}\left(\boldsymbol{S}^{(n)}\right)^{-1} \boldsymbol{\beta}^{(n)}}{\langle n+1 \mid n+1\rangle} .
\end{aligned}
$$

[1] H. Hergert, S. Bogner, T. Morris, A. Schwenk, and K. Tsukiyama, The in-medium similarity renormalization group: A novel ab initio method for nuclei, Phys. Rep. 621, 165 (2016).

[2] H. Hergert, In-medium similarity renormalization group for closed and open-shell nuclei, Physica Scripta 92, 023002 (2016).

[3] J. M. Yao, B. Bally, J. Engel, R. Wirth, T. R. Rodríguez, and $\mathrm{H}$. Hergert, $\mathrm{Ab}$ initio treatment of collective correlations and the neutrinoless double beta decay of ${ }^{48} \mathrm{Ca}$, Phys. Rev. Lett. 124, 232501 (2020).

[4] A. Belley, C. G. Payne, S. R. Stroberg, T. Miyagi, and J. D. Holt, $\mathrm{Ab}$ initio neutrinoless double-beta decay matrix elements for ${ }^{48} \mathrm{Ca},{ }^{76} \mathrm{Ge}$, and ${ }^{82} \mathrm{Se}$, Phys. Rev. Lett. 126, 042502 (2021).

[5] D. L. Hill and J. A. Wheeler, Nuclear constitution and the interpretation of fission phenomena, Phys. Rev. 89, 1102 (1953).

[6] J. M. Yao, J. Engel, L. J. Wang, C. F. Jiao, and H. Hergert, Generator-coordinate reference states for spectra and $0 \nu \beta \beta$ decay in the in-medium similarity renormalization group, Phys. Rev. C 98, 054311 (2018).

[7] T. R. Rodríguez and G. Martínez-Pinedo, Energy density functional study of nuclear matrix elements for neutrinoless $\beta \beta$ decay, Phys. Rev. Lett. 105, 252503 (2010).

[8] N. L. Vaquero, T. R. Rodríguez, and J. L. Egido, Shape and pairing fluctuations effects on neutrinoless double beta decay nuclear matrix elements, Phys. Rev. Lett. 111, 142501 (2013)

[9] L. S. Song, J. M. Yao, P. Ring, and J. Meng, Relativistic description of nuclear matrix elements in neutrinoless double- $\beta$ decay, Phys. Rev. C 90, 054309 (2014)

[10] J. M. Yao, L. S. Song, K. Hagino, P. Ring, and J. Meng, Systematic study of nuclear matrix elements in neutrinoless double$\beta$ decay with a beyond-mean-field covariant density functional theory, Phys. Rev. C 91, 024316 (2015)

[11] C. F. Jiao, J. Engel, and J. D. Holt, Neutrinoless double- $\beta$ decay matrix elements in large shell-model spaces with the generator- coordinate method, Phys. Rev. C 96, 054310 (2017).

[12] N. Hinohara and J. Engel, Proton-neutron pairing amplitude as a generator coordinate for double- $\beta$ decay, Phys. Rev. C 90, 031301(R) (2014).

[13] J. Menéndez, N. Hinohara, J. Engel, G. Martínez-Pinedo, and T. R. Rodríguez, Testing the importance of collective correlations in neutrinoless $\beta \beta$ decay, Phys. Rev. C 93, 014305 (2016).

[14] Y. Toh, C. J. Chiara, E. A. McCutchan, W. B. Walters, R. V. F. Janssens, M. P. Carpenter, S. Zhu, R. Broda, B. Fornal, B. P. Kay, et al., Evidence for rigid triaxial deformation at low energy in ${ }^{76} \mathrm{Ge}$, Phys. Rev. C 87, 041304(R) (2013).

[15] T. R. Rodríguez, Role of triaxiality in ${ }^{76} \mathrm{Ge}$ and ${ }^{76} \mathrm{Se}$ nuclei studied with gogny energy density functionals, J. Phys. G 44, 034002 (2017).

[16] S. Shinohara, H. Ohta, T. Nakatsukasa, and K. Yabana, Configuration mixing calculation for complete low-lying spectra with a mean-field hamiltonian, Phys. Rev.C 74, 054315 (2006).

[17] J. Engel and J. Menéndez, Status and future of nuclear matrix elements for neutrinoless double-beta decay: a review, Rep. Prog. Phys. 80, 046301 (2017).

[18] P. Ring and P. Schuck, The nuclear many-body problem (Springer Science \& Business Media, 2004).

[19] J. Menéndez, A. Poves, E. Caurier, and F. Nowacki, Novel nuclear structure aspects of the $o v \beta \beta$-decay, Journal of Physics: Conference Series 267, 012058 (2011)

[20] C. W. Johnson, W. E. Ormand, and P. G. Krastev, Factorization in large-scale many-body calculations, Computer Physics Communications 184, 2761 (2013).

[21] C. W. Johnson, W. E. Ormand, K. S. McElvain, and H. Shan, Bigstick: A flexible configuration-interaction shell-model code, arXiv preprint arXiv:1801.08432 (2018).

[22] J. Menendez, A. Poves, E. Caurier, and F. Nowacki, Disassembling the nuclear matrix elements of the neutrinoless $\beta \beta$ decay, Nucl. Phys. A 818, 139 (2009). 
[23] B. Bally, A. Sánchez-Fernández, and T. R. Rodríguez, Variational approximations to exact solutions in shell-model valence spaces: Calcium isotopes in the p f shell, Phys. Rev. C 100, 044308 (2019).

[24] B. Bally, A. Sánchez-Fernández, and T. R. Rodríguez, Symmetry-projected variational calculations with the numerical suite taurus, Eur. Phys. J. A 57, 1 (2021).

[25] V. Srivastava, A unified view of the orthogonalization methods, Journal of Physics A: Mathematical and General 33, 6219 (2000)

[26] D. R. Entem and R. Machleidt, Accurate charge-dependent nucleon-nucleon potential at fourth order of chiral perturbation theory, Phys. Rev. C 68, 041001(R) (2003)

[27] K. Hebeler, S. K. Bogner, R. J. Furnstahl, A. Nogga, and A. Schwenk, Improved nuclear matter calculations from chiral low-momentum interactions, Phys. Rev. C 83, 031301(R) (2011)

[28] J. M. Yao, A. Belley, R. Wirth, T. Miyagi, C. G. Payne, S. R. Stroberg, H. Hergert, and J. D. Holt, Ab initio benchmarks of neutrinoless double- $\beta$ decay in light nuclei with a chiral hamiltonian, Phys. Rev. C 103, 014315 (2021) 